\title{
Reliability Enhancement of Modular Multilevel Converter by Applying Fault Tolerant Control
}

\author{
Mahmoud Abdelsalam, Hatem Diab, Sarath Tennakoon and Alison Griffiths \\ School of electrical engineering \\ Staffordshire university \\ Stoke on Trent, United Kingdom \\ mahmoud.abdelsalam@research.staffs.ac.uk
}

\begin{abstract}
Modular Multilevel Converters (MMC) are considered very suitable for the transmission of bulk power. Increasing the reliability of MMCs forms a real challenge since they are built from series connection of sub-modules. Applying the concept of fault tolerance to the MMC control system can significantly increase the reliability of the MMC as it will be available in normal as well as faulty conditions. This paper presents an analysis of the MMC reliability enhancement gained from applying different fault tolerant control techniques. The reliability is investigated through solving differential equations generated from Markov chains.
\end{abstract}

Keywords—Markov chains; fault tolerant control; MMC.

\section{INTRODUCTION}

The fault tolerant control (FTC) system is the system which is able to perform its desired control functions at high level of stability and security during the occurrence of a certain fault. FTC systems are gaining the interest of many researchers in different research fields because of their ability to enhance the overall reliability of systems. FTC systems are classified into two main types: passive and active [1-3].

As shown in Fig. 1, active FTC system receives the continuous fault status from the FDU. Then at the fault condition, it re-configures the control system to a new system which isolates the faulty part of the system and maintains the stability of the plant. It is important to mention that the redesign process of the system is performed online which normally may allow some changes to desired control functions or degradation in the performance [4-5].

The main disadvantage of active FTC techniques is the slow response of them due to the time consumed on the fault detection and controller redesign. However, the main advantage of these techniques is their ability to detect wide range of faults [5].

In passive FTC, the controller is designed in the offline mode then it operates with fixed behavior during normal and abnormal conditions. Therefore, it should be designed such that it can tolerate the faults [6-7]. The main advantages of passive FTC techniques can be summarized in the following points:
- There is no need for the fault detection process as the system is working with the same parameters during normal as well as fault conditions.

- The response of them is very fast due to the elimination of FDU and the control redesign processes.

However, it is very difficult for one passive FTC system to detect different types of faults since they are designed in the offline mode [8].

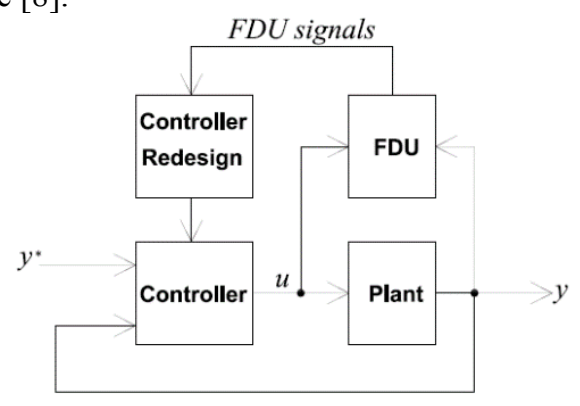

Fig. 1: Structure of active FTC.

According to the literature, there are different types of submodule faults that can strike MMCs and affect their operation [9-10]. Since MMCs are used in important applications and at the same time they form a big investment, it is extremely important to detect these fault very quickly to protect the whole system from sudden failures. For this particular purpose, the sub-module faults are investigated in the following subsections. Moreover, popular fault detection techniques are discussed to find out the advantages and disadvantages of each method and pointing what is needed in the process of fault detection of MMCs.

MMCs should work at high level of reliability as they usually work in critical applications that do not have the luxury of losing the power transfer. Based on this fact, it is highly recommended to provide the control of the MMC with fault tolerance capability to make the MMC able from delivering the desired power during faulty conditions without the need to block the converter [11].

This paper presents an investigation of MMC reliability enhancement when two different fault tolerant control 
techniques are used. The first technique is based on using redundant sub-modules while the second depends on modifying the control of the MMC during faults. Section II presents the operation concept of the MMC and its mathematical model. The reliability analysis using Markov chains of the MMC is illustrated in section III. Section IV shows the conclusion and major findings of the presented work.

\section{Modular Multilevel CONVERTERS}

\section{A. The structure of MMCs}

The MMC consists of sub-modules connected in series forming a leg in each phase. The sub-module can be a halfbridge or a full-bridge and each sub-module has a capacitor that buffers the energy from the dc to the ac side and vice versa; thus the dc-link capacitor is not required. A high-speed bypass switch is added to the output port of the sub-module to isolate the sub-module in case of a fault. As shown in Fig. 2, each phase leg is divided into two arms upper and lower. Each arm has identical numbers of sub-modules to generate balanced voltages in the two arms of each phase. Inductors are installed in arms to smooth and filter the currents [12-13].
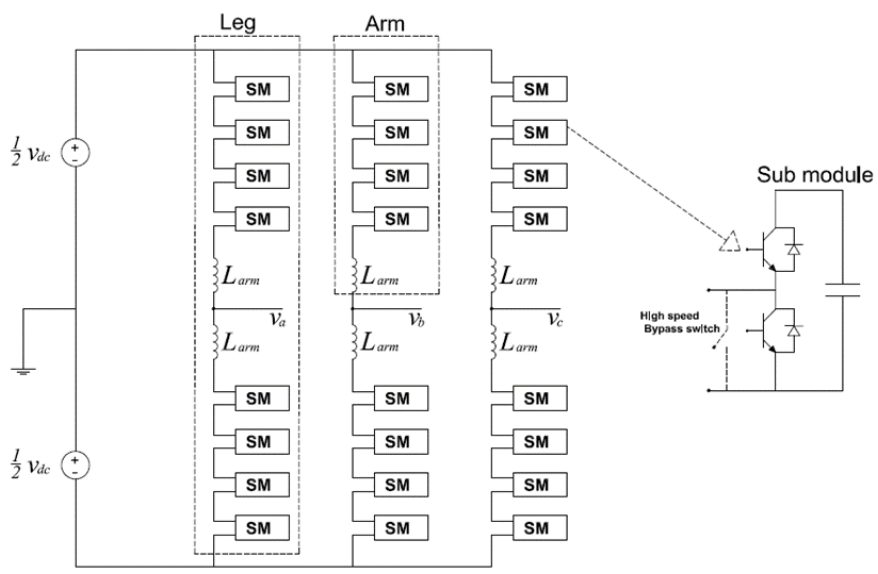

Fig. 2. The structure of the MMC.

\section{B. MMC Operating Modes}

The MMC half bridge sub-module has four operation modes during which energy is transferred:

Mode 1: When S1 is closed, S2 is opened, and the arm has positive polarity. The current flows into the capacitor, charging it. The sub-module is then inserted. See Fig. 3(a).

Mode 2: When $\mathrm{S} 1$ is opened, $\mathrm{S} 2$ is closed, and the arm has positive polarity. The sub-module is bypassed, and the current flows towards the next sub-module, keeping the capacitor charge constant. The submodule is then bypassed. See Fig. 3(b).

Mode 3: $\mathrm{S} 1$ is closed, $\mathrm{S} 2$ is opened, and the arm has negative polarity. The capacitor starts to discharge and then the sub-module is inserted. See Fig. 3(c).

Mode 4: When S1 is opened, S2 is closed, and the arm has negative polarity. The current flows towards the next module, keeping the capacitor charge constant, after which and the sub-module is bypassed. See Fig. 3(d).

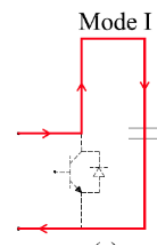

(a)

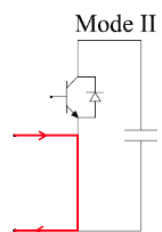

(b)

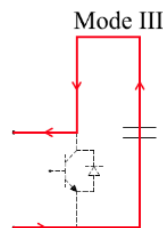

(c)

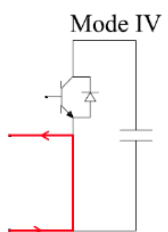

(d)
Fig. 3. Different operating modes of MMC sub-modules. (a) Mode 1. (b) Mode 2. (c) Mode 3. (d) Mode 4.

\section{Mathematical Model of MMC}

For the purpose of designing an inner control system for the $\mathrm{MMC}$, it is necessary to mathematically analyze the converter. The converter arms, shown in Fig. 4, are represented as variable capacitors connected in a series with arm resistance and inductance. The number of sub-modules per arm and the switching frequency is assumed as infinite to simplify the analysis because of the perfect sinusoidal output voltage and total voltage balancing between arms. The arm capacitance insertion is represented with a modulation value $m_{\text {arm } x}$, where $x$ may be $u$ for upper arm or $l$ for lower arm. The $m_{\text {arm } x}$ is varied from 0 to 1 . For example, when $m_{\text {arm } u}=0$, this indicates that all sub-modules in the upper arm are bypassed and when $m_{\text {arm } l}=1$, this indicates that all sub-modules in the lower arm are inserted. The value $\Sigma v_{C x}$ is the sum of arm capacitor voltages. The arm voltage is given by:

$v_{x}(t)=m_{\text {arm } x}(t) . \Sigma v_{C x}$.

The inserted arm capacitance is given by:

$C_{\text {insx }}=\frac{C_{S M}}{N \cdot m_{\text {arm } x}(t)}$

where $C_{S M}$ is the capacitance of one sub-module and $N$ is the number of sub-modules per arm. If the arm current is $i_{x}(t)$, the total capacitor voltage dynamics can be expressed by:

$\frac{d \Sigma v_{C x}}{d t}=\frac{i_{x}(t)}{C_{\text {ins } x}}$.

Substituting (2) in (3), the capacitor voltage dynamics can be expressed for the upper and lower arms by:

$\frac{d \Sigma v_{C u}}{d t}=\frac{N \cdot m_{\text {arm } u} \cdot i_{u}}{C_{S M}}$

$\frac{d \Sigma v_{C l}}{d t}=\frac{N \cdot m_{\text {arm } l} \cdot i_{l}}{C_{S M}}$

The current $i_{\text {diff }}$ is the differential current, which circulates between the phase legs. The circulating current of any phase is described as follows:

$i_{\text {diff }}=\frac{i_{u}+i_{l}}{2}$.

By performing the necessary circuit analysis, the output phase voltage $v_{o}$ is given by:

$v_{o}=\frac{v_{d c}}{2}-L_{\text {arm }} \frac{d i_{u}}{d t}-m_{\text {arm } u} \Sigma v_{C u}$

$v_{o}=-\frac{v_{d c}}{2}+L_{a r m} \frac{d i_{l}}{d t}+m_{\text {arm } l} \Sigma v_{C l}$. 


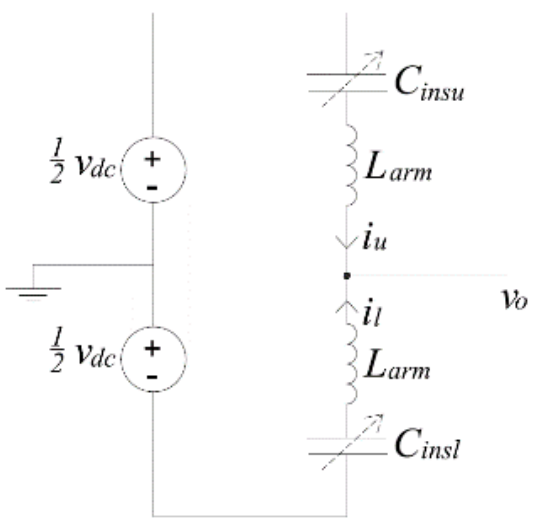

Fig. 4. The MMC average model.

\section{MMC RELIABILITY ANALYSIS}

A system failure can be represented by its probability to occur; this is done considering the process behavior as a random variable which receive its value from finite state space elements. To assess the reliability of the MMC, firstly the failure rate of the IGBT $\lambda_{0}$ should be assumed. The reliability function $R(t)$ is the probability of success for the system which is expressed as:

$R(t)=1-F(t)$

Where $F(t)$ is the probability of the system failure which is complementary to the $R(t)$ and is given by:

$F(t)=\int_{0}^{t} f(\tau) d \tau$

Where $f(t)$ is rate of change for the failure probability of a certain components, thus (10) can be rewritten as:

$f(t)=\frac{d F(t)}{d t}$

Another important definition is the mean time to failure (MTTF) which gives an indication for the expected life time of a component that cannot be repaired or maintained, the MTTF is expressed as:

$M T T F=\int_{0}^{\infty} R(t) d t$

For a system without a fault tolerant control, the MMC will fail if one sub-module suffers from a fault in either the upper or lower legs. To model the reliability of the converter in this case, a Markov chain is developed as shown in Fig. 5.

In this case the failure rate for one sub-module in a certain leg should equal:

$\lambda_{1}=(2 N-1) \lambda_{0}$

Where $\lambda_{0}$ is the failure rate of each IGBT switch.

Based on the Markov chain and from (13) substituting in (11), this gives:

$\frac{d P_{1}}{d t}=-2 \lambda_{1} P_{1}(t)$

Solving the first order differential equation presented in (10), the reliability function can be expressed as:

$R(t)=P_{1}(t)=e^{-2(2 N-1) \lambda_{0} t}$

From (15) substituting in (12), the mean time to failure is:

MTTF $F_{M M C}$ without FTC $=\frac{1}{2 \lambda_{0}}\left(\frac{1}{(2 N-1)}\right)$

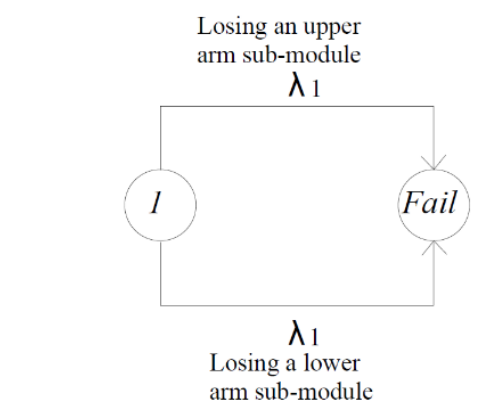

Fig. 5. Markov chain of the MMC without FTC.

\section{MMC RELIABILITY ENHANCMENT USING FTC}

The enhancement of the MMC reliability is calculated while applying two FTC techniques. The first technique achieves the fault tolerance using redundant sub-modules while the second depends on modifying the control of the MMC without using redundant sub-modules.

\section{A. FTC using redundant sub-modules}

Regarding tolerating the fault using redundant sub-modules, the idea depends on disconnecting the faulty sub-module and inserting a new redundant sub-module without the disconnection of the MMC as shown in Fig. 6 [13-15].

The process of insertion of the redundant sub-modules is done using a strict control. At each triggering time, the control system identifies the state of each sub-module by the aid of a suitable fault detection algorithm. If any sub-module is subjected to high switching commutations or a fault, the control system bypasses the faulty sub-module and replaces it by a redundant sub-module [15].

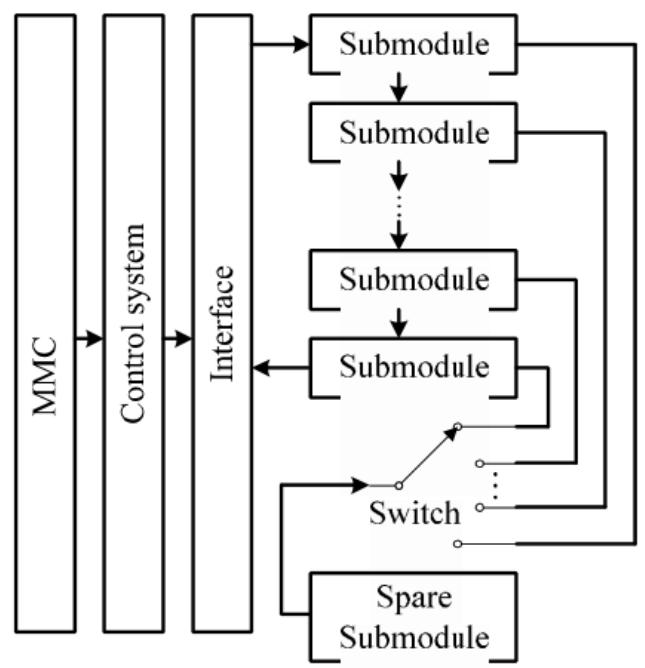

Fig. 6: Structure of MMC with redundant sub-modules.

In this case, the reliability of the MMC can be figured out from Fig. 7 which has two failure rates. The first failure rate when losing one sub-module, $\lambda_{1}$. While the second failure rate, $\lambda_{2}$ indicates losing two sub-modules in the same leg. In the submodule redundant based FTC, each leg is assumed to be 
equipped with one redundant sub-module. Thus, the submodule failure rates are given by:

$\lambda_{1}=\left(\frac{2 N-1}{2}+1\right) \lambda_{0}$

$\lambda_{2}=\left(\frac{2 N-1}{2}\right) \lambda_{0}$

According to Markov chain, the failure probability of the first state is:

$\frac{d P_{1}}{d t}=-\lambda_{1} P_{1}(t)$

Solving the differential equation given in (19) gives:

$P_{1}(t)=e^{-\left(\frac{2 N-1}{2}+1\right) \lambda_{0} t}$

Following the same way, the failure probability of state 2 gives:

$\frac{d P_{1}}{d t}=\lambda_{1} P_{1}(t)-\lambda_{2} P_{2}(t)$

Solving (21) gives:

$P_{2}(t)=\left(\frac{2 N-1}{2}\right)\left(e^{-\left(\frac{2 N-1}{2}\right) \lambda_{0} t}-e^{-\left(\frac{2 N-1}{2}+1\right) \lambda_{0} t}\right)$

From (20) and (22), the probability of the fail state is the summation of $P_{1}(t)$ and $P_{2}(t)$ :

$R(t)=P_{1}(t)+P_{2}(t)=\left(\left(\frac{1-2 N}{2}\right) e^{-\left(\frac{2 N-1}{2}+1\right) \lambda_{0} t}\right)+$ $\left(\frac{2 N-1}{2}\right)\left(e^{-\left(\frac{2 N-1}{2}\right) \lambda_{0} t}\right)$

From (23) substituting in (12), the MTTF is:

$M T T F_{M M C \text { with FTC } 1}=\frac{1}{\lambda_{0}}\left(\frac{1-N}{N+1)}\right)$

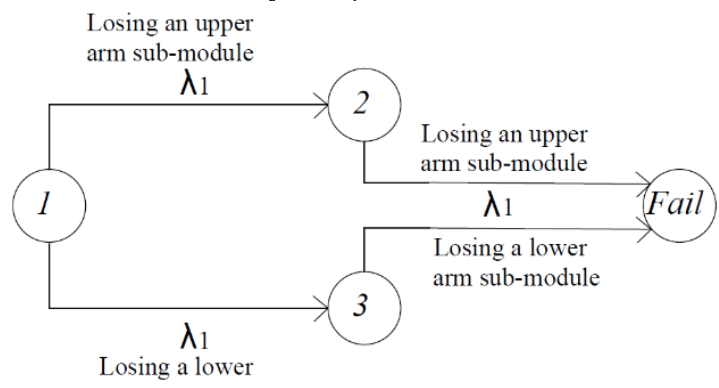

Fig. 7. Markov chain of the MMC with the first FTC technique.

\section{B. FTC based on modifing the MMC inner control}

Unlike the previous technique, the MMC is not equipped by redundant sub-modules. The MMC inner control modifies the control and isolates the faulty sub-modules. To simplify the calculations and give a sense for the results, the reliability calculations will be performed for the simulation model which incorporates four sub-modules per arm. In this particular case, the Markov reliability chain is developed as shown in Fig. 8. The Markov reliability chain shows that the MMC with the proposed FTCU have four possible finite states before reaching the failure state instead of one as shown in the previous section. The possible states are:

- $\quad$ State 1: The healthy state (no faulty sub-modules).

- State 2: Losing a sub-module in the upper arm.

- State 3: Losing a sub-module in the lower arm.

- State 4: Losing two sub-modules (one in the upper arm and one in the lower arm).

- Fail state: Losing two sub-modules in the upper arm or two sub-modules in the lower arm or both.
In this case, two failure rates should be presented $\lambda_{1}$ and $\lambda_{2} \cdot \lambda_{1}$ is the failure rate of one sub-module and will be the same as calculated in (13). $\lambda_{2}$ is the failure rate for losing two submodules and should equal:

$\lambda_{2}=(2 N-3) \lambda_{0}$

Based on the Markov chain, the probability for the first state $P 1$ will remain the same as presented in (15). Regarding the probability of the second state $P 2$, it can be expressed as follows:

$\frac{d P_{2}}{d t}=-\left(\lambda_{1}+\lambda_{2}\right) P_{2}(t)+\lambda_{1} P_{1}(t)$

From (26) substituting in (11), the solution of the differential equation gives:

$P_{2}(t)=\frac{2 N-3}{2}\left(e^{-\left(\lambda_{1}+\lambda_{2}\right) t}-e^{-2 \lambda_{1} t}\right)$

$P_{3}(t)$ has the same probability of $P_{2}(t)$ since the conditions are the same.

Moving to the forth state probability, it is also can be emphasized from the Markov chain as follows:

$\frac{d P_{4}}{d t}=-\left(2 \lambda_{2}\right) P_{4}(t)+\lambda_{1} P_{2}(t)+\lambda_{1} P_{3}(t)$

From (28) and substituting in (11), the solution of this differential equation is expressed as:

$P_{4}(t)=\frac{(2 N-3)^{2}}{4}\left(e^{-2 \lambda_{1} t}+e^{-2 \lambda_{2} t}\right)-\frac{(2 N-3)^{2}}{2} e^{-\left(\lambda_{1}+\lambda_{2}\right) t}$

The reliability function $R(t)$ is the summation of the four probabilities as follows

$R(t)=P_{1}(t)+P_{2}(t)+P_{3}(t)+P_{4}(t)$

$=\left((2 N-2)+\frac{(N-3)^{2}}{4}\right) e^{-2(2 N-1) \lambda_{0} t}+((2 N-3)-$

$\left.\frac{(2 N-3)^{2}}{4}\right) e^{-2(2 N-2) \lambda_{0} t}+\left(\frac{(2 N-3)^{2}}{4}\right) e^{-2(2 N-3) \lambda_{0} t}$

From (6.18) substituting in (6.8), the mean time to failure is:

$M T T F_{M M C}$ with FTC $=\frac{1}{2 \lambda_{0}}\left(\frac{\left((2 N-2)+\frac{(2 N-3)^{2}}{4}\right)}{(2 N-1)}+\right.$

$\left.\frac{\left((2 N-3)-\frac{(2 N-3)^{2}}{4}\right)}{(2 N-2)}+\frac{\left(\frac{(2 N-3)^{2}}{4}\right)}{(2 N-3)}\right)$

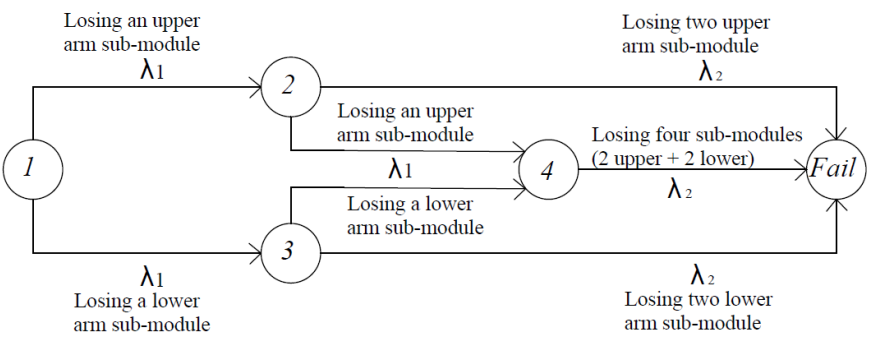

Fig. 8. Markov chain of the MMC with the second FTC technique.

\section{RESUlts AND DiscusSiONS}

To compare between the reliability before and after the application of the two fault tolerant control strategies, the reliability density functions presented in (15), (23) and (30) are plotted against time for five level MMC. The IGBT failure rate $\lambda_{0}$ is assumed to be $10^{-7} h^{-1}$ which is almost three years. 
As shown in Fig. 9, the reliability density function is considerably enhanced which means that the rate of failure for the MMC has been decreased. This is also clearly appearing in table I where the MTTF has significantly increased when applying the two FTC strategies.

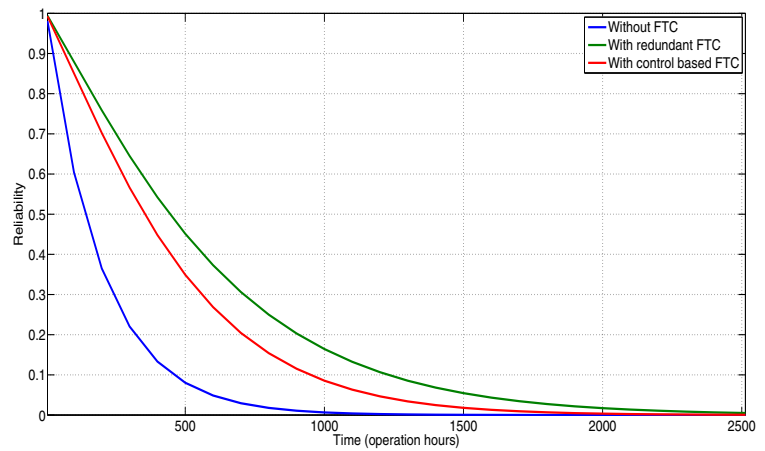

Fig.9: The reliability of the MMC using different FTC techniques.

TABLE I

COMPARISON BETWEEN MTTF ASSOCIATED WITH DIFFERENT FTC TECHNIQUES

\begin{tabular}{ll}
\hline \multicolumn{1}{c}{ CONDITION } & \multicolumn{1}{c}{ MTTF (IN HOURS) } \\
\hline Without FTC & 714285 \\
With redundancy FTC & 8750000 \\
With control based FTC & 163958335 \\
\hline \hline
\end{tabular}

\section{CONCLUSION}

In this paper, the reliability of the MMC has been carefully studied and analized using Markov chains. The effect of using FTC on the MMC is investigated. Results showed that using the FTC concept will significantly increase the MTTF of the MMC which means that the MMC will be very reliable since it is availability has increased to contain normal and faulty operation conditions.

\section{REFERENCES}

[1] M. Blanke, R. Izadi-Zamanabadi, S. A. Bogh, and Z. P. Lunau. Fault tolerant control systems-a holistic view. Control Engineering Practice, 5(5):693$702,1997$.

[2] M. Blanke, M. Staroswiecki, and N. E. Wu. Concepts and methods in faulttolerant control. In American Control Conference, volume 4, pages 26062620, 2001.

[3] M. Blanke, M. Kinnaert, J. Lunze, and M. Staroswiecki. Diagnosis and Fault-Tolerant Control. Springer-Verlag, 2006.

[4] K. Tsuda, D. Mignone, G. Ferrari-Trecate, and M. Morari. Reconfiguration strategies for hybrid systems. In American Control Conference, volume 2, pages $867-873,2001$.

[5] Y. Zhang and J. Jiang. Bibliographical review on reconfigurable fault tolerant control systems. Annual Reviews in Control, 32(2):229-252, 2008.

[6] G. H. Yang, Zhang S. Y., J. Lam, and J.L. Wang. Reliable control using redundant controllers. IEEE Transactions on Automatic Control, 43(11):1588-1593, Nov 1998.

[7] R.J. Veillette. Reliable linear-quadratic state-feedback control. Automatica, 31(1):137-144, 1995.

[9] G.H. Yang, J.L. Wang, Y.C. Soh, and K.Y. Lou. Reliable state feedback control synthesis for uncertain linear systems. Asian Journal of Control, 5(2):301-308, 2003.

[10] E. Wolfgang, \Examples for failures in power electronics systems," in presented at ECPE Tutorial Reliability of Power Electronic Systems., Nuremberg, Germany, Apr. 2007.

[11] K. Fischer, T. Stalin, H. Ramberg, T. Thiringer, J. Wenske, and R. Karlsson, Investigation of converter failure in wind turbines," in Elforsk report, Nov. 2012.

[12] J. Qin, \& M. Saeedifard, "Predictive control of a modular multilevel converter for a back-to-back HVDC system", IEEE Transactions on Power Delivery, vol 27 no. 3, 1538-1547, 2012.

[13] S. Debnath, J. Qin, B. Bahrani, M. Saeedifardand and P. Barbosa, "Operation, Control, and Applications of the Modular Multilevel Converter: A Review", IEEE transactions on power electronics, vol. 30, no. 1, January 2015.

[14] W. Zhang, D.Xu, P. N. Enjeti, H. Li, J. T. Hawke, \& H. S. Krishnamoorthy, "Survey on fault-tolerant techniques for power electronic converters", IEEE Transactions on Power Electronics, 2014.

[15] Son, G. T., Lee, H. J., Nam, T. S., Chung, Y. H., Lee, U. H., Baek, S. T., ... \& Park, J. W. (2012). Design and control of a modular multilevel HVDC converter with redundant power modules for noninterruptible energy transfer.Power Delivery, IEEE Transactions on, 27(3), 1611-1619. 